\title{
Das numerische Verhältnis der Geschlechter in der Vogelwelt.
}

\author{
Von Friedrich von Lucanus, Berlin.
}

Bezüglich des numerischen Verhältnisses der Geschlechter in der Vogelwelt ist allgemein die Ansicht verbreitet, dab eine Uberzahl männlicher Individuen vorhanden sei, und man führt mit einem gewissen Recht als Beweis hierfür die Balzspiele, den Sängerkrieg und die Kämpfe der Männchen in der Fortpflanzungszeit an.

Diesen Standpunkt vertritt auch LrEbe in seiner Arbeit: "Die Überzahl der Männchen" (Ornithologische Monatsschrift 1894) und versucht ihn durch die Ergebnisse seiner Erfahrungen, die er bei der Aufzucht junger dem Nest entnommener Vögel machte, zu rechtfertigen. So weist er daranf hin, dab er in den Bruten von Coccothraustes coccothraustes. I., Fringilla coelebs L., Galerida cristata L., Emberiza citrinella L., Erithacus rubeculus L. sowie der Thurdiden, besonders von Turdus merulı L. hänfig mehr Männchen als Weibchen gefunden habe.

Besonders interessant ist Liebe's Angabe über das Sexualverhältnis von Passer clomesticus L. Ais Futter für seine Raubvögel fing er in der näheren Unngehung seines Wohnhauses öfters eine größere Anzahl Sperlinge ein, wobei sich bezüglich des Geschlechts folgende Zahlenverhältnisse, ergaben: „Im Frühjalrr 1892 11 Stück, worunter nur 2 Weibchen, im Frühjahr 1893 20 Stïck und zwar lediglich Mämnchen, im Herbst 189322 Stiick, worunter 1 Weibchen, im Winter $189+$ bis zum Februar 4 Männchen.

Im Gegensatz hierzu liegen nun in neuerer Zeit eine ganze Reihe von Beobachtungen vor; die gerade auf ein umgekehrtes Verhältnis der Geschlechter, nämlich auf einen Überschuß an Weibchen hindeuten.

Eine diesbezïgliche, selır interessante Mitteilung bringt die Deutsche Jägerzeitung in Nr. 51 des Jahrgangs 1915 unter der Überschrift: „Rascher Weibchenersatz beim Sperber". Der Verfasser des Artikels R. Hönning schoß am 11. 5. 1911 ein Sperberweichen am Horst. $\mathrm{Zn}$ dem übriggebliebenen Männchen gesellte sich sofort ein neutes Weibchen, das Hörnivri gleichfalls erlegte. Am 21. 5. beobachtete der Schütze auf demselben Horst ein drittes Weibchen beim Legen eines Eies, das er auch abschoß. 
Es war ein in der Mauser befindliches Stück im Jugendkleid, welches zugleich ein Beweis dafür ist; daß die Fortpflanzungsfähigkeit der Vögel unter Umständen schon vor dem Anlegen des Alterskleides eintreten kann.

Dieser zweimalige schnelle Weibchenersatz bei Accipiter nisus L. zeigt, daß die Annahme von einem Uberschuß der Männcheu keineswegs immer zutrifft und nicht ohne weiteres verallgemeinert werden darf.

Für eine Mehrzal des weiblichen Geschlechts beim Sperber sprechen auch folgende Erscheinungen: Von einem Forstbeamten erhielt ich einst 3 am Uhu erbeutete Sperber, unter denen sich 2 Weibchen befanden. Ich selbst schoß gelègentlich meiner Vogelzugstudien in Rossitten innerhalb einer Stunde 3 Sperber, von denen ebenfalls 2 weiblich waren und erlegte auf einem Pürschgang Mitte Mai ein altes Sperberweibchen, das anscheinend ungepaart war, da in der betreffenden Gegend kein Männchen festgestellt werden konnte.

Nicht allein für den Sperber, sondern auch für andere Raubvogelarten liegen Beobachtungen vor, die einen ÜberschuB an Weibchen bestätigen. So fügt Hörning am Schluß seiner Mitteilung hinzu, dab er auch bei WVanderfalkenpaaren dieselbe Erfahrung machen konnte, daB ein abgeschossenes Weibchen bald ersetzt wurde. Über einen gleichen, äußerst interessanten Fall berichtet Hнinвoтн in den Ornithologischen Monatsberichten $\mathrm{Nr} .9$ des Jahrgangs 1912 in folgender Weise:

.Herr Frifenich Falz-Fein in Ascania Nova, Südrußland, hat in seinem Park eine Menge grober Nistkästen aufgehängt, die für Braut- und Mandarinenten bestimmt sind, aber auch gern von Rötel- und Turmfalken in Besitz genommen werden. Aus einer solchen Höhle wollte Herr FaLz-F lax das Rötelfalkenpaar vertreiben und glaubte dies am besten dadurch tun zu können, daß er das Weibchen abschoß. Nach kurzer Zeit kam der Witwer mit einem neuen Weibchen an und in wenigen Stunden erlegte der Gutsherr 8 Rötelfalkenweibchen an dem Nistkasten, bis er schließlich, um der Sache ein Ende zu machen, auch den männlichen Vogel beseitigte. Dieses Männchen hatte gar keine Schwierigkeiten bei der Erlangung einer neuen Gattin, es flog in die Luft, rief ein paarmal und kehrte mit einem neuen Weibchen heim."

Alle diese Erfahrungen lassen vermuten, daß bei den Raubvögeln das weibliche Geschlecht erheblich zahlreicher vertreten ist als das männliche, und daß die übliche Anschauung von einer Uberzahl der Männchen für die Gattungen Falco und Accipiter jedenfalls nicht zutrifft.

Aber nicht nur für die Raubvögel, sondern auch für andere Vögel lassen sich Fälle anführen, die zu der Auffassung von einer Mehrzahl der Männchen im Widerspruch stehen. So berichtet HeINRoth in seiner oben erwähnten Arbeit, daß er schon 
wiederholt im Berliner zoologischen Garten Weibchen von Turdus merula L., Phoenicurus phoenicurus L. und Muscicapa atricapilla L. ohne Gatten brütend angetroffen habe. In allen diesen Fällen wohnten die einzelnen Vogelweibchen in der Nähe von richtigen Paaren und sind offenbar in der Höhe der Brunst auf eine Paarungsaufforderung ihrerseits hin von den fremden Männchen getreten worden.

Für meine Studien über die Erblichkeit des Vogelgesangs zog ich junge Amseln (Turdus merula L.), Nachtigallen (Luscinia megarynchos $\mathrm{BR}_{\mathrm{R}}$ ) und Hänflinge (Acanthis cannabina $\mathrm{L}$.) auf. Unter vier jungen Amselı und vier jungen Nachtigallen, die je einem Nest entstammten, befanden sich je 2 Weibchen, und drei Nestgeschwister von Acanthis cannabina waren sämtlich weiblichen Geschlechts. Eine Brut junger Kleinspechte (Dendrocopus minor L.), welche ich bei einem Vogelhändler fand, enthielt 2 Weïbchen und 1 Männchen.

Dr. Hernooth hatte die Liebenswürdigkeit, mir seine 'lagebücher, die er über die Aufzucht junger Vögel führt, zur Einsicht $z \mathfrak{u}$ überlassen. Seine Aufzeichnungeu enthalten folgende Angaben über das Geschlechtsverhältnis in 10 aus der Freiheit stammenden, vollzähligen Bruten:

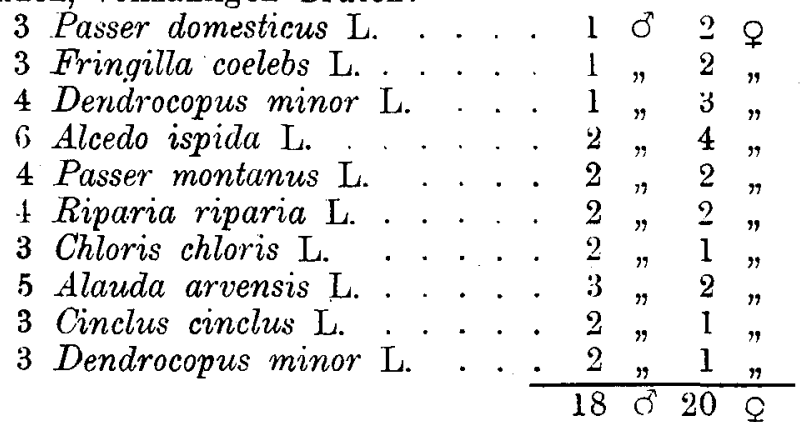

Die vorher erwähnten 4. Bruten bestehen zusammen aus $50^{\circ} 0^{\circ}$ und 9 으, so daß sich also insgesamt ein Geschlechtsverhältnis von $2: 30^{\circ} 0^{\circ} \mathrm{zu} 29$ oᄋ ergibt.

In diesen 14 Bruten findet sich nur in 4 Fällen eine Mehrzahl an Männchen, dagegen in 5 Fällen eine Mehrzahl an Weibchen und einmal sogar eine nur aus Weibchen bestehende Nachkommenschaft, während in \pm Fällen die Anzahl der Geschlechter eine gleiche ist.

Dies Ergebnis ist insofern beachtenswert, als es die bisher vertretene Ansicht von einer Überzahl der Männchen nicht rechtfertigt, sondern ebenso wie die Raubvogelbeobachtungen gerade im Gegenteil auf eine Mehrzahl an Weibchen hinweist.

Im Gegensatz zu allen diesen Erscheinungen, die für einen Überschuß an Weibchen sprechen, konnte ich einst im Oktober in Braunlage im Harz eine Beobachtung machen, in der es sich um eine auffallende Überzahl an Männchen handelt. 
Die reifen Beeren der im Ort stehenden Ebereschen übten anf die Gimpel eine große Anziehungskraft aus, die sich hier täglich in großer Menge einfanden. Es war die kleine mitteleuropäische Form Pyrrhula pyrrhula europaea VIEILL, die im Harz liäufiger Brutvogel ist. Sehr auffallend war die große Zahl der Männchen. Auf etwa 6-8 Männchen kam nur ein Weibchen.

Das ungleiche Verhältnis der Geschlechter bei den zur Herbstzeit zusammengescharten Gimpeln erwähuen auch $N_{\text {AUMANN }}$ and FrIDERICH. Nach NAUMANN macht sich eine Über\%ahl der Weibchen am häufigsten bemerkbar, während nach FRIDERICH bald die Männchen, bald die Weibchen vorherrschell. Interessant ist, daß die Angabe NaUManv's zugleich wieder für ein allgemein häufigeres Vorkommen der Weibchen spricht.

Von einer eigentlichen Trennung der Geschlechter auf dem Zuge, wie bei Fringilla coelebs L. kann man bei Pyrrhula pyrrhula L. jedenfalls nicht sprechen, da eine Vermischung der Geschlechter, wenn auch im ungleichen Verhältnis, stattfindet.

Friderich meint, dab die einzelnen Familien sich zusammenfinden, bleibt aber eine Erklärung für das ungleiche und wechselnde Verhältnis der Geschlechter schuldig.

Bei den von mir in Braunlage beobachteten Gimpeln fiel mir auf, daß bei weitaus die meisten Männchen noch im Federwechsel waren und noch erhebliche Spuren des Jugendkleides zeigten, während die Weibchen fast sämtlich das völlig vermauserte Alterskleid trugen. Die mausernden jungen Männchen konnten in Anbetracht der späten Jahreszeit, es war Ende Oktober, nur aus der zweiten Brut stammen, während die Weibchen in Hinsicht anf ihren bereits vollendeten Gefiederwechsel entweder alte Stücke oder Junge aus der ersten Brut sein mußten.

Man kann aus dieser Erscheinung den Schlub ziehen, daß in den zweiten Bruten bedeutend mehr Männchen als Weibchen erbrütet worden sind.

Die oben erwähnten jungen Vögel, die Hегмкотн und ich aufzogen, wurden mit Ausnahme der Wasserstare und Lurchen im Friihjahr dem Nest entnommen, entstammten also der ersten Brut. Wie wir gesehen haben, war hier das weibliche Geschlecht erheblich zahlreicher vertreten als das männliche.

Die jungen Wasserstare und Feldlerchen waren im Juli erbrïtet worden, gehörten daher der zweiten Brut an. Das Geschlechtsverhältnis der ersteren beträgt $20^{\circ}: 1 \%$, das der letzteren $3 \delta^{2}: 2 \wp \emptyset$. Wir haben also hier analog meiner bei Pyrrhula gemachten Beobachtung zwei weitere Fälle von einer Mehrzahl der Männchen unter den Jungen aus der zweiten Brut.

Das Ergebnis dieser Erscheinungen legt die Vermutung nahe, dab vielleicht in den ersten Bruten im allgemeinen vorwiegend 
Weibchen, in den späteren Bruten dagegen vorherrschend Männclien erzeugt werden.

Aus der Physiologie wissen wir, daß die Geschlechtsbestimmung des Embryo keineswegs eine willkürliche ist, sondern $\mathrm{daB}$ sie bestimmten Gesetzen unterliegt, die natürlich für die Frage nach dem Sexualverhältnis der Vögel von großer Bedeutung sind and daher hier berücksichtigt werden müssen.

Der Wiener Mediziner LEopoud Schenk gründete bekanntlich seine Lehre vom künstlichen Einfluß auf die Geschlechtsbestimmung beim Menschen auf das Prinzip der Ernährungsweise und Körperbeschaffenheit der Mutter, indem er annahm, daß die geschlechtsbestimmenden Reize allein in der Eizelle enthalten seien, und daß die Samenzelle lediglich individuelle Eigenschaften und Artmerkmale, aber nicht das Geschlecht auf den Embryo vererbe. Durch Veränderung des Eiweiß- und Zuckergehalts im Körper des Weibes glaubte Schenk die Geschlechtsbestimmung beeinflussen zu können.

Die Schenk'sche Theorie ist durch die neueren Forschmngen auf dem Gebiete der Ontogenie widerlegt worden, die nachgewiesen haben, daß bei geschlechtlicher Fortptlanzung eiue ovariale Geschlechtsbestimmung nicht zutrifft, sondern daß beide Eltern ihren EintluB ausüben.

Sowohl die männlichen, wie die weiblichen Keimzellen tragen die Anlagen und Merkmale für beide Geschlechter in sich. Infolge Verschmelzung der beiden Keimzellen bei der Befruchtung müssen dalıer in jedem Embryo viererlei Anlagekomplexe, nämlich 2 für das weibliche und '2 für das mämnliche Geschlecht vorhanden sein. Die Bestimmung des Geschlechts erfolgt nun dadurch, daß entweder die männlichen oder die weiblichen Anlagen gefördert werden, die Entwicklung der entgegengesetzten Charaktere aber unterdrückt wird, je nachdem die eine oder andere geschlechtliche Tendenz stärker hervortritt.

Die Träger der Eigenschaften der Eltern sind die in den Spermatogonien und Ovogonien enthaltenen Chromosomen, die gemeinsam den Aufbau des neu entstehenden Individuums vollziehen, indem sie die Artmerkmale anf den Nachkommen übertragen.

Die Vererbung des Geschlechts erfolgt du'ch besondere Geschlechtschromosomen, die aus männlichen und weiblichen Elementen bestehen.

Die nelleste Forschung auf diesem Gebiet hat nun ergeben, daß die Samenzelle ein Geschlechtschromosom weniger besitzt als die Eizelle, und da die Chromosomen parweis auftreten, so hat also das weibliche Geschlecht stets eine gerade Chromosomenzahl, das männliche dagegen eine ungerade, indem hier bei einem Geschlechtschromosomenpaar der Partner fehlt.

Wir sehen hieraus, daß das weibliche Geschlecht reicher an Materie ist als das männliche, und man darf daher wohl 
annehmen, daß auch zu seiner Erzeugung mehr Kraft und Stoff gehört als zur Bildung des männlichen Geschlechts.

Einen Beweis für die Richtigkeit dieser Annahme liefert uns die Fortpflanzungsgeschichte der Insekten.

Die männlichen Hymenopteren werden nur parthogenetisch erzeugt, die weiblichen dagegen gehen nur aus befruchteten Eiern hervor. Thre Entwicklung beansprucht also die doppelte Chromosomenzahl als der Aufbau des mänulichen Organismus.

Bei Apis mellifica L. genïgt aber nicht die Befruchtung des Eies allein zur Bildung eines vollwertigen Weibchens, sonderu die Larve entwickelt sich nur dann zur Königin, wenn sie während ihres Wachstums besonders gut und kräftig ernährt wird, während im anderen Falle nur Weibchen mit verkümmerten Geschlechtsorganen, die sogenannten Arbeiterinnen, entstehen. Wenn dies anch mit der eigentlichen Geschlechtsbestimmung nicht unmittelbar zusammenhängt, da ja diese bereits durch die Befruchtung erfolgte, und daher nicht epigame, sondern syngame Vermehrung vorliegt, so erscheint es doch insofern beachtenswert, als daraus hervorgeht, wie sehr das weibliche Geschlecht zu seiner Entwicklung einer erhöhten Zufuhr von Kraft bedarf.

Sehr interessant und lehrreich sind die Versuche, die Russow in -jüngster Zeit für seine Studien über das Sexualverhältnis ausgeführt hat. Es gelang ihm beim Kaninchen durch Verabreichung von Lecithin an weibliche Tiere das Geschlechtsverhältnis der Nachkommen zugunsten der weiblichen Form zu verändern. Zehn von denselben Männchen belegte Weibchen warfen ohne vorhergegangene Lecithinbehandlung 36 männliche und 29 weibliche Junge, nach der Anwendung von Lecithin dagegen 26 Männchen und 40 Weibchen. Die weibliche Tendenz in den Ovogenien ist also durch das Lecithin gefördert worden.

Bei der Zucht buntfarbiger Wäuse machte ich die Erfahrung, daB anfangs das numerische Verhältnis der Geschlechter mit einem geringen Überschuß weiblicher Tiere fast ein gleiches war. 20 Würfe von verschiedenen Elterntieren ergaben zusammen 38 Männchen und 41 Weibchen. Als später infolge engster Inzucht die Mäuse anfingen zu degenerieren, was sich in verlangsamtem Wachstum, geringerer Körpergröße, die mitunter geradezu in Zwergform ausartete, und zarter Körperkonstitution offenbarte, trat unter den Jungen eine erhebliche Mehrzahl des männlichen Geschlechts auf. 5 Würfe vou stark degenerierten Eltern bestanden zusammen aus 16 männlichen und nur 11 weiblichen . Iungen.

Eine schwächliche gelb- und weißgescheckte Maus warf nach der Paarung mit einem nah verwandten gleichfalls schwachen, zimmetfarbenen Männchen 4 männliche und 2 weibliche Junge, dagegen nach der Paarung mit einem wilden Hausmausmännchen 2 männliche und 2 weibliche Junge. Eine sehr degenerierte schokoladenfarbig and weißgescheckte Maus brachte 
nach Befruchtung durch dasselbe wilde Hausmausmännchen 1 männliches und :3 weibliche Junge zur Welt. In beiden Fällen tritt also unter der Nachkommenschaft eine Zunahme des männlichen Geschlechts auf, deren Ursache wohl auf das bessere und kräftigere Spermatozoon der wilden Stammform zurïckzuführen ist.

Die Ergebnisse dieser Versuche deuten darauf hin, dab offenbar nicht nur die Anzahl der Geschlechtschromosomen, sondern auch ihre Beschaffenheit für den Geschlechtscharakter des Embryo von Bedeutung ist. Eine bessere Qualität der Chromosomen, die vielleicht in einer reichhaltigeren Menge von Chromatin besteht, begünstigt anscheinénd die weibliche Tendenz in den Keimzellen, eine geringere Qualität dagegen die männliche Veranlagung.

Da aber die Beschaffenheit der Chromosomen mit der jeweiligen Körperkonstitution ihres Besitzers zusammenhängt, so mu $B$ auch dieser einen Anteil an der Geschlechtsbestimmung zufallen, wie es sich ja auch in den Experimenten mit den degenerierten Mäusen und den mit. Lecithin behandelten Kaninchen gezeigt hat. So erscheint also der weibliche Organismus im Vergleich zum männlichen als das Produkt größerer Kraftentfaltung, was ja auch dadurch, daß die Samenzelle ein Geschlechtschromosom weniger besitzt als die Eizelle, zum Ausdruck kommt.

Bei den Vögeln steht zu Beginn der Brutzeit der Fortpflanzungstrieb und die Entwicklung der Geschlechtsorgane auf dem Höhepunkt, der ganze Körper befindet sich in vollster Kraft. Infolgedessen wird die weibliche T'endenz in den Keimzellen überwiegen.

Bei den späteren Bruten dagegen ist der Organismus der Eltern durch das vorangegangene Fortpflanzungsgeschäft, die Brutzeit und die mühsame Aufzucht der Jungen, durch welche die eigene Ernährung beeinträchtigt wurde, erlieblich geschwächt. Dieser Verlust an Lebensenergie wird sich altch in der Beschaffenheit der Geschlechtschromosomen bemerkbar machen, die hierdurch herabgesetzt ist und daher die männliche Tendenz in den Keimzellen begïnstigt.

Die Vermutung: „in den ersten Gelegen mehr Weibchen, in den späteren Gelegen mehr Männchen", die ich auf Grund der im ersten Teil meiner Arbeit angeführten Beobachtungen aus dem Leben der Vögel aussprach, scheint also vom physiologischen Standpunkt aus betrachtet, nicht unberechtigt zu sein.

Dieser Grundsatz darf freilich nicht so aufgefaßt werden, dab in jeder ersten Brut immer das weibliche, in jeder späteren stets das männliche Geschlecht vorherrschen muB, sondern es kann sich natürlich nur um einen allgemeinen Durchschnitt handeln, da ja die individuelle Körperbeschaffenheit und Veranlagung der einzelnen Elterntiere verschieden ist.

Wenn in den ersten Gelegen vorwiegend Weibchen, in den späteren dagegen mehr Männchen erbrïtet werden, so muß bei 
allen den Vogelarten, die 2 Bruten jährlich machen, im allgemeinen das Verhältnis der Geschlechter ein gleiches sein. Eine erhebliche Ungleichheit der Geschlechter wird sich zeitweise nur dann geltend machen, wenn durch ungünstige Witterungsverhältnisse entweder im Frühjahr die erste, oder im Sommer die zweite Brut zum größten Teil verloren ging.

Die obell erwähnte Angabe Friderichs, dab in manchen Jahren die Anzahl der Gimpelmännchen, in anderen wieder die der Weibchen bedeutend überwiegt, würde auf diese Weise zu erklären sein.

Bei Vogelarten, die mehr als 2 Bruten hintereinander machen, mïßte eine bedeutende Uberzahl an Männchen vorhanden sein. Daß dies tatsächlich der Fall ist, beweist der Haussperling, der ;-4 mal im Laufe des Sommer's zur Fortptlanzung schreitet, und bei dem das männliche Geschlecht ganz erheblich stärker vertreten ist als das weibliche; denn Bilder, wo 5, 6 oder noch mehr Männchen in heftiger Fehde um ein .Weibchen bemüht sind, kanı man im Frühjahı und Sommer täglich auf den Straßen beobachten. Auch aus der anfangs erwälınten Angabe LiEBE's über das Sexualverhältnis von Passer domesticus geht ja deutlich hervor, wie außergewöhnlich grob die Zahl der männlichen Sperlinge ist.

Bei allen Vögeln, die jährlich nur einmal brüten, müßte nach dem oben aufgestellten Grundsatze eine Mehl\%ahl von Weibchen in Frscheinung treten. Für die Richtigkeit einer solchen Annahme spricht der' wiederholt beobachtete, übelraschend schnelle Weibchenersat; beim Wanderfalken, Rötelfalken und Sperber, die alle nur eime Nachkommenschaft im .Jahr großziehen.

Zum SchluB unserer Betrachtung wollen wir noch einen Blick darauf werten, welche Erfahrungen aus der Kanarienund Gefiügelzucht über das Sexualverhältnis vorliegen.

In der Literatur über die Kanarienzucht finden sich zahlreiche Klagen über die geringe Anzahl der erbrüteten Männchen. Interessant ist, daß diese Angaben zu der sonst üblichen Anschauung von einer Mehrzahl der Männchen zunächst gleichfalls in Widerspruch stehen. Andererseits darf man den Erfahrungen, die bei der Zucht gefangener Vögel gesammelt werden, keine allzu große Bedeutung beimessen. Es ist hier zunächst in Betracht zu ziehen, daß viele Junge durch Eingriffe des Züchters, sowie durch mangelhafte Pflege seitens der alten Vögel bereits in den Nestern zugrunde gehen, so daß das Endergebnis kein richtiges Bild von der Anzahl der Geschlechter in den einzelnen Brutell ergibt. Ferner ist zu berücksichtigen, daß die Verhältnisse in der Gefangenschaft wesentlich anders sind als in der Freiheit. Die Kanarienhähne werden mit Rücksicht auf ihre Gesangsausbildung in kleinen, verdunkelten Käfigen gehalten. Die Weibchen werden häufig aus Sparsamkeitsgründen in kalten Räumen bei einfachem Köruerfutter überwintert. Unter 
solchen ungünstigen und unnatürlichen Lebensbedingungen verbrauchen die Vögel einen unverbältnismäßig großen Teil ilhrer Kraft zur Erhaltung des Lebens und stehen daher zu Beginn der Fortpflanzungszeit nicht auf dem physischen Höhepunkt. Die Folge davon ist, daB in der ersten Brut nicht, wie es unter normalen Verhältnissen der Fall wäre, meln Weibchen, sondern gerade im Gegenteil vielleicht mehr. Männchen erzeugt werden. Während der Fortpllanzungszeit wird den Vögeln zur Aufzucht ihrer Jungen reichlich Eifutter gereicht. Durch dies unnatürliche Kraftfutter wird aber der Vérlust an Lebensenergie, den die Elternvögel in der erstell Fortpflanzungsperiode erlitten haben, nicht nur ersetzt, sondern die physische Beschaffenheit wird sogar über ein natürliches Mab gesteigert. So ist es nicht unwahrscheinlich, daß infolge des günstigen Körperzustandes der Zuchttiere aus den späteren Bruten vorwiegend weibliche Nachkommen hervorgehen. Tatsächlich herrscht denn anch in den Kreisen der Züchter vielfach die Auffassung, daß in den ersten Gelegen hauptsächlich Männchen, in den späteren dagegen mehr Weibchen erbrütet werden.

Da im allgemeinen :3 Bruten von einem Kanarienpaar im Laufe des Sommers erzielt werden, so ist es ans den dargelegten Gründen selır wahrscheinlich, daß die Mehrzahl der Jungen der 2. und 3. Brut weiblichen Geschlechts ist, woraus als Endergebnis eine Überzahl der Weibchen hervorgehen würde. Die Klage der Zü̈chter über die geringe Anzahl der erbrüteten Männchen scheint also nicht ganz unberechtigt zu sein.

Ebenso wie bei der Kauarienzucht liegen auch bei der Geflügelzucht die Verhältnisse wesentlich anders als draußen in der Natur. Hier hat der Mensch durch künstliche Zuchtwahl neue Tierformen geschaffen, deren Organismus durch besonder's angezüchtete Eigeuschaften, wie schnelle und große Mastfähigkeit, hohe Eierproduktion und eigenartige Körperformen eine bedeutende Veränderung erfahren hat, die zweifellos auch die Fortpflanzung und alle mit dieser zusammenhängenden Erscheinungen erbeblich beeinflußt. Dazu kommt noch, daß die Züchter durch besondere Fütterung und Wartung häufig in das Sexualleben der Tiere künstlich eingreifen. Die Ergebnisse, die in bezug auf die Fortpflanzung aus der Geflügelzacht hervorgehen, leiden also an dem Nachteil, daß ihnen bedentende Fehlerquellen zugrunde liegen, die bei einem Vergleich mit den Verhältnissen in der Natur berïcksichtigt werden müssen, wo die Tiere unter ganz anderen Bedingungen leben, und daher auch ganz andere biologische Grundsätze zur Geltung kommen.

In meiner Arbeit lag es mir in erster Linie daran, zu zeigen, daß die alte Anschauung von einer Überzahl des männlichen Geschlechts unter den Vögeln, die in der Literatur immer wieder angegeben wird, ais allgemeiner Grundsatz keine Gültigkeit haben kann. Wie ich nachgewiesen habe, läßt sich 
bedentendes Material dafür anführen, daß keineswegs immer die Männchen, sondern in Gegenteil häufig die Weibchen in größerer Anzabl vertreten sind.

Wenn ich versucht habe, hierfür eine Erklärung zu finden, so beanspruche ich keineswegs, eine endgültige Lösung dieser Frage gegeben zu haben, denn in dem überaus schwierigen Problem von der Vererbung und Bestimmung des Geschlechts ist noch vieles unaufgeklärt, worüber uns erst die weiteren Fortschritte auf dem Geliet der Ontogenie Aufschluß geben werden. Mir kam es vielmehr darauf an, in meinem Beitrag für diese Festschrift anf eine Erscheinung im Leben der Vögel hinzuweisen, die erst wenig beachtet worden ist, und sie unter einem anderen, neuen Gesichtspunkt zu betrachten, in der Hoffnung hierdurch zu weiterer Forschung in der Frage nach dem numerischen Verhältnis der Geschlechter in der Vogelwelt anzuregen.

\section{Literaturverzeichnis :}

NaUmann, Naturgeschichte der Vögel Mitteleuropas, 1905.

FriderTCE, Naturgeschichte der deutschen Vögel, 1905.

Ornithologische Monatsberichte, Jahrgang 1912.

Ornithologische Monatssehrift, Jabrgang 1894.

Deutsche Jägerzeitung, Jahrgang 1915.

CorRens-GoldschMidT, Die Vererbung und Bestimunung des Geschlechts, 1913.

K roNaCHER, Grundzüge der Züchtungsbiologie, 1912.

SCHENK, Einfluß auf das Geschlechtsveräältnis 1898. 